Research Article

\title{
The Range of the Spectral Projection Associated with the Dunkl Laplacian
}

\author{
Salem Ben Said ${ }^{1}{ }^{1}$ and Hatem Mejjaoli ${ }^{2}$ \\ ${ }^{1}$ Department of Mathematical Sciences, College of Science, United Arab Emirates University, Al Ain Abu Dhabi, UAE \\ ${ }^{2}$ Department of Mathematics, College of Science, Taibah University, Al Madinah AL Munawarah, Saudi Arabia
}

Correspondence should be addressed to Salem Ben Said; salem.bensaid@uaeu.ac.ae

Received 24 October 2019; Accepted 27 April 2020; Published 24 July 2020

Academic Editor: Mitsuru Sugimoto

Copyright (c) 2020 Salem Ben Said and Hatem Mejjaoli. This is an open access article distributed under the Creative Commons Attribution License, which permits unrestricted use, distribution, and reproduction in any medium, provided the original work is properly cited.

For $s \in \mathbb{R}$, denote by $P_{k}^{s} f$ the "projection" of a function $f$ in $\mathscr{D}\left(\mathbb{R}^{d}\right)$ into the eigenspaces of the Dunkl Laplacian $\Delta_{k}$ corresponding to the eigenvalue $-s^{2}$. The parameter $k$ comes from Dunkl's theory of differential-difference operators. We shall characterize the range of $P_{k}^{s}$ on the space of functions $f \in \mathscr{D}\left(\mathbb{R}^{d}\right)$ supported inside the closed ball $\overline{B(O, R)}$. As an application, we provide a spectral version of the Paley-Wiener theorem for the Dunkl transform.

\section{Introduction}

Analysis of the Dunkl Laplacian operator $\Delta_{k}$ on $\mathbb{R}^{d}$ commenced in the early 90's, inspired by numerous results in the Euclidean setting, as well as some extensions of this to flat symmetric spaces. Here, the parameter $k$ comes from Dunkl's theory of differential-difference operators [1]. In recent years, there have been increasing interests in the study of problems involving the Dunkl Laplacian and have received a lot of attention, see for instance [1-15]. The purpose of this paper is to study a family of eigenfunctions for the Dunkl Laplacian derived through the use of the inversion formula for the Dunkl transform. Our main result may be interpreted as a contribution to the spectral theory of the Dunkl Laplacian.

To state our main result, we need to introduce some notation. Writing the inversion formula for the Dunkl transform in polar coordinates, we obtain

$$
f(x)=\int_{0}^{\infty} s^{2 \lambda_{k}+1} f_{k}^{s}(x) d s, f \in \mathscr{D}\left(\mathbb{R}^{d}\right),
$$

where $f_{k}^{s}$ are "projections" of $f$ into the eigenspaces of $\Delta_{k}$ corresponding to the eigenvalue $-s^{2}$ (see (35)). We may also write the projection operators $f \mapsto f_{k}^{s}$ as Dunkl-convolution with a normalized Bessel function of the first kind (see (43)). In this paper, we discuss on $\mathscr{D}\left(\mathbb{R}^{d}\right)$ how properties of $f$ are related to properties of the eigenfunctions $f_{k}^{s}$. Essentially, we prove a theorem characterizing $f_{k}^{s}$ for $f \in \mathscr{D}\left(\mathbb{R}^{d}\right)$ with $\operatorname{supp}(f) \subset \overline{B(O, R)}$, involving analytic continuation to $s \in \mathbb{C}$ and growth estimates of type, for all $N \in \mathbb{N}$ and for all multi-index $m \in \mathbb{N}^{d}$,

$$
\left|\partial_{x}^{m} f_{k}^{s}(x)\right| \leq C_{k, m, N}(\|x\|)(1+|s|)^{-N} e^{(R+\|x\|)|\operatorname{Im} s|}, x \in \mathbb{R}^{d}
$$

where $C_{k, m, N}$ is a positive continuous increasing function on $\mathbb{R}_{+}$(see Theorem 9). Several contributions have been dedicated to this subject, see for instance [16-22].

As an application of the main result, we prove a spectral version of the complex Paley-Wiener theorem for the Dunkl transform $\mathscr{F}_{k}$ given in [23]. More precisely, we characterize the set of functions $\varphi(s, \eta)$ defined on $\mathbb{R} \times \mathbb{S}^{d-1}$ for which there exists a compactly supported smooth function $f$ with support in $\overline{B(O, R)}$ so that $\varphi(s, \eta)=\mathscr{F}_{k}(f)(s \eta)$ (see Theorem 10). 


\section{Background}

For $x, y \in \mathbb{R}^{d}$, we let $\langle x, y\rangle$ denote the usual Euclidean inner product of $\mathbb{R}^{d}$ and $\|x\|:=\sqrt{\langle x, x\rangle}$ the Euclidean norm. Let $\mathbb{S}^{d-1}$ be the unit sphere in $\mathbb{R}^{d}$. We denote by $d \sigma$ as the Lebesgue surface measure on $\mathbb{S}^{d-1}$.

For a nonzero vector $\alpha \in \mathbb{R}^{d}$ define the reflection $r_{\alpha}$ by

$$
r_{\alpha}(x):=x-2\left(\langle\alpha, x\rangle /\|\alpha\|^{2}\right) \alpha, x \in \mathbb{R}^{d} .
$$

A root system is a finite set $\mathscr{R}$ of nonzero vectors in $\mathbb{R}^{d}$ such that $\alpha, \beta \in \mathscr{R}$ implies $r_{\alpha}(\beta) \in \mathscr{R}$. If, in addition, $\alpha, \beta \in$ $\mathscr{R}$ and $\alpha=c \beta$ for some scalar $c$ implies $c= \pm 1$, then $\mathscr{R}$ is called reduced. Henceforth, we will assume that $\mathscr{R}$ is a reduced root system. Fix a set of positive roots $\mathscr{R}^{+}$, so that $\mathscr{R}=\mathscr{R}^{+} \cup\left(-\mathscr{R}^{+}\right)$.

The finite reflection group $G$ generated by the root system $\mathscr{R}$ is the subgroup of the orthogonal group $O(d)$ generated by the reflections $\left\{r_{\alpha}: \alpha \in \mathscr{R}^{+}\right\}$.

For a given root system $\mathscr{R}$, a multiplicity function $k: \mathscr{R} \longrightarrow \mathbb{R}_{+} ; \alpha \mapsto k_{\alpha}$ is a nonnegative $G$-invariant function.

Given a reduced root system $\mathscr{R}$ on $\mathbb{R}^{d}$ and a multiplicity function $k=\left(k_{\alpha}\right)_{\alpha \in \mathscr{R}}$, we define the weight function $\vartheta_{k}$ by

$$
\vartheta_{k}(x):=\prod_{\alpha \in \mathscr{R}^{+}}|\langle\alpha, x\rangle|^{2 k_{\alpha}}, x \in \mathbb{R}^{d} .
$$

Then, $\vartheta_{k}$ is a positively homogeneous $G$-invariant function of degree $2\langle k\rangle$, where

$$
\langle k\rangle:=\sum_{\alpha \in \mathscr{R}^{+}} k_{\alpha} .
$$

The main ingredient of the Dunkl theory is a family of commuting first-order differential-difference operators, $T_{\xi}(k)$ (called the Dunkl operators [1]), defined by

$T_{\xi}(k) f(x):=\partial_{\xi} f(x)+\sum_{\alpha \in \mathscr{R}^{+}} k_{\alpha}\left\{\left(f(x)-f\left(r_{\alpha} x\right)\right) /\langle\alpha, x\rangle\right\}\langle\alpha, \xi\rangle, \xi \in \mathbb{R}^{d}$,

where $\partial_{\xi}$ is the ordinary partial derivative with respect to $\xi$. The Dunkl operators are akin to the partial derivatives and they can be used to define the Dunkl Laplacian $\Delta_{k}$, which plays the role similar to that of the ordinary Laplacian $\Delta$,

$$
\begin{aligned}
\Delta_{k} f(x):= & \sum_{i=1}^{d} T_{\xi_{i}}(k)^{2} f(x)=\Delta f(x)+2 \sum_{\alpha \in \mathscr{R}^{+}} k_{\alpha}\{\langle\nabla f(x), \alpha\rangle /\langle\alpha, \mathrm{x}\rangle\} \\
& -\sum_{\alpha \in \mathscr{R}^{+}} k_{\alpha}\left\{\left(f(x)-f\left(r_{\alpha} x\right)\right) /\langle\alpha, x\rangle^{2}\right\}\|\alpha\|^{2},
\end{aligned}
$$

where $\left\{\xi_{1}, \cdots, \xi_{d}\right\}$ is an orthonormal basis of $\left(\mathbb{R}^{d},\langle\cdot, \cdot\rangle\right)$. The above explicit expression of $\Delta_{k}$ has been proved in [24].
For arbitrary finite reflection group $G$, and for any nonnegative multiplicity function $k$, there is a unique linear operator $V_{k}$ on the space of algebraic polynomials on $\mathbb{R}^{d}$ that intertwines between the Dunkl operators and the partial derivatives,

$$
T_{\xi}(k) V_{k}=V_{k} \partial_{\xi}, \forall \xi \in \mathbb{R}^{d}, V_{k} 1=1
$$

It has been proved in [25] that $V_{k}$ has a Laplace type representation which allows to extend $V_{k}$ to larger function spaces:

$$
V_{k} f(x)=\int_{\mathbb{R}^{d}} f(\xi) d \mu_{x}^{k}(\xi)
$$

with a unique probability measure $\mu_{x}^{k} \in M^{1}\left(\mathbb{R}^{d}\right)$. In fact, $V_{k}$ induces a homeomorphism of $C\left(\mathbb{R}^{d}\right)$ and also that of $C^{\infty}\left(\mathbb{R}^{d}\right)$; see $[23,26]$.

For $x, y \in \mathbb{R}^{d}$, define

$$
E_{k}(x, y):=V_{k}\left(e^{\langle\cdot, y\rangle}\right)(x)
$$

For fixed $y$, the function $E_{k}(\cdot, y)$ is the unique realanalytic solution of $T_{\xi}(k) f(x)=\langle y, \xi\rangle f(x)$ with $f(0)=1$ (see $[13,27])$. Further, the (Dunkl) kernel $E_{k}$ has a unique holomorphic extension to $\mathbb{C}^{d} \times \mathbb{C}^{d}$ and satisfies the following properties:

Fact 1 (see, for instance, $[11,25]$ ).

(1) For all $z, w \in \mathbb{C}^{d}$ and $\lambda \in \mathbb{C}$, we have $E_{k}(z, w)=E_{k}$ $(w, z)$ and $E_{k}(\lambda z, w)=E_{k}(z, \lambda w)$.

(2) For every multi-index $m \in \mathbb{N}^{d}$, we have

$$
\left|\partial_{z}^{m} E_{k}(x, z)\right| \leq\|x\|^{|m|} e^{\|x\|\|\operatorname{Re} z\|},
$$

where $|m|=m_{1}+\cdots+m_{d}$. In particular, $\left|E_{k}(x, i y)\right| \leq 1$ for all $x, y \in \mathbb{R}^{d}$. by

For $f \in L^{1}\left(\mathbb{R}^{d}, \vartheta_{k}(x) d x\right)$, the Dunkl transform is defined

$$
\mathscr{F}_{k} f(\xi):=c_{k}^{-1} \int_{\mathbb{R}^{d}} f(x) E_{k}(x,-i \xi) \vartheta_{k}(x) d x, \quad \xi \in \mathbb{R}^{d}
$$

where $c_{k}$ is the constant

$$
c_{k}:=\int_{\mathbb{R}^{d}} e^{-\|x\|^{2} / 2} \vartheta_{k}(x) d x .
$$

The Dunkl transform was introduced in [28] where the $L^{2}$-isometry (or the Plancherel theorem) was proved, while the main results of the $L^{1}$-theory were established in [11]. In particular, it has been proved that if $f$ and $\mathscr{F}_{k} f$ are in $L^{1}$ 
$\left(\mathbb{R}^{d}, \vartheta_{k}(x) d x\right)$, then for almost every $x \in \mathbb{R}^{d}$,

$$
f(x)=c_{k}^{-1} \int_{\mathbb{R}^{d}} \mathscr{F}_{k} f(\xi) E_{k}(\xi, i x) \vartheta_{k}(\xi) d \xi, \xi \in \mathbb{R}^{d}
$$

It is worth mentioning that the Dunkl transform is a homeomorphism of the Schwartz space $\mathcal{S}\left(\mathbb{R}^{d}\right)$. Further, according to ([29], Proposition 5.7.8), for $f \in L^{1}\left(\mathbb{R}^{d}, \vartheta_{k}(x) d\right.$ $x)$ such that $f(x)=f_{0}(\|x\|)$ with $f_{0}: \mathbb{R}_{+} \longrightarrow \mathbb{C}$, we have

$$
\mathscr{F}_{k} f(\xi)=\mathscr{H}_{\lambda_{k}} f_{0}(\|\xi\|)
$$

where

$$
\lambda_{k}:=\langle k\rangle+(d-2) / 2
$$

and $\mathscr{H}_{\alpha}$ is the Hankel transform of index $\alpha$ on $L^{1}\left(\mathbb{R}_{+}, r^{2 \alpha+1} d r\right)$, given by

$$
\mathscr{H}_{\alpha} g(s)=1 /\left(2^{\alpha} \Gamma(\alpha+1)\right) \int_{0}^{\infty} g(r) j_{\alpha}(r s) r^{2 \alpha+1} d r .
$$

Here, $j_{\alpha}$ is the normalized Bessel function of the first kind defined by

$$
\begin{aligned}
& j_{\alpha}(z):=\Gamma(\alpha+1)(z / 2)^{-\alpha} \mathbb{J}_{\alpha}(z), \quad \text { where } \\
& \mathbb{J}_{\alpha}(z)=(z / 2)^{\alpha} \sum_{n=0}^{\infty}\left\{(-1)^{n}(z / 2)^{2 n}\right\} /\{n ! \Gamma(\alpha+n+1)\} .
\end{aligned}
$$

Let $y \in \mathbb{R}^{d}$ be given. For $f \in \mathcal{S}\left(\mathbb{R}^{d}\right)$, the generalized translation operator is defined by

$$
\tau_{y} f(x):=c_{k}^{-1} \int_{\mathbb{R}^{d}} \mathscr{F}_{k} f(\xi) E_{k}(i x, \xi) E_{k}(i y, \xi) \vartheta_{k}(\xi) d \xi, x \in \mathbb{R}^{d}
$$

Fact 2 (see [26]). The translation operator has the following properties:

(1) For all $x, y \in \mathbb{R}^{d}, \tau_{y} f(x)=\tau_{x} f(y)$.

(2) For fixed $y \in \mathbb{R}^{d}, \tau_{y}$ extends to a continuous linear mapping from $C^{\infty}\left(\mathbb{R}^{d}\right)$ into itself

(3) If $f, g \in \mathcal{S}\left(\mathbb{R}^{d}\right)$ and $y \in \mathbb{R}^{d}$, then

$$
\int_{\mathbb{R}^{d}} \tau_{y} f(x) g(x) \vartheta_{k}(x) d x=\int_{\mathbb{R}^{d}} f(x) \tau_{-y} g(x) \vartheta_{k}(x) d x .
$$

The generalized translation operator is used to define a convolution structure: For $f, g \in \mathcal{S}\left(\mathbb{R}^{d}\right)$,

$$
f *_{k} g(x):=c_{k}^{-1} \int_{\mathbb{R}^{d}} f(y) \tau_{x} \breve{g}(y) \vartheta_{k}(y) d y,
$$

where $\breve{g}(x):=g(-x)$. We can also write the convolution $*_{k}$ as

$$
f *_{k} g(x)=c_{k}^{-1} \int_{\mathbb{R}^{d}} \mathscr{F}_{k} f(\xi) \mathscr{F}_{k} g(\xi) E_{k}(i x, \xi) \vartheta_{k}(\xi) d \xi
$$

We refer the reader to [15] for more details on the convolution product $*_{k}$. It is worth mentioning, for the distributional version of the Dunkl transform, the translation operator and the Dunkl convolution of distributions and properties, we refer the reader to [30].

For $n \in \mathbb{N}$, let $\mathscr{H}_{k}^{n}$ be the space of $k$-harmonic polynomials of degree $n$ on $\mathbb{R}^{d}$,

$$
\mathscr{H}_{k}^{n}=\operatorname{Ker} \Delta_{k} \cap \mathscr{P}_{n}\left(\mathbb{R}^{d}\right)
$$

where $\Delta_{k}$ is the Dunkl Laplacian and $\mathscr{P}_{n}\left(\mathbb{R}^{d}\right)$ denotes the space of homogeneous polynomials of degree $n$ on $\mathbb{R}^{d}$. The restriction of elements in $\mathscr{H}_{k}^{n}$ on the unit sphere $\mathbb{S}^{d-1}$ in $\mathbb{R}^{d}$ are the so-called $k$-spherical harmonics. We shall not distinguish between $Y_{k}^{n} \in \mathscr{H}_{k}^{n}$ and its restriction to $\mathbb{S}^{d-1}$. The space $\mathscr{H}_{k}^{n}$ has a reproducing kernel $Q_{k}^{n}(\cdot, \cdot)$ in the sense that

$$
f(x)=d_{k}^{-1} \int_{\mathbb{S}^{d-1}} f(y) Q_{k}^{n}(x, y) \vartheta_{k}(y) d \sigma(y), \forall f \in \mathscr{H}_{k}^{n},\|x\| \leq 1
$$

Here, $d_{k}$ is the constant

$$
d_{k}:=\int_{\mathbb{S}^{d-1}} \vartheta_{k}(x) d \sigma(x)=c_{k} /\left\{2^{\lambda_{k}} \Gamma\left(\lambda_{k}+1\right)\right\},
$$

where $c_{k}$ and $\lambda_{k}$ are as defined in (13) and (16), respectively. According to [31], for $x, y \neq 0$, the kernel $Q_{k}^{n}$ can be written as

$$
Q_{k}^{n}(x, y)=(\|x\|\|y\|)^{n}\left\{\left(n+\lambda_{k}\right) / \lambda_{k}\right\} V_{k}\left[C_{n}^{\lambda_{k}}(\langle\cdot, y /\|y\|\rangle)\right](x /\|x\|),
$$

where $V_{k}$ is the Dunkl intertwining operator (8), and $C_{n}^{\alpha}$ is the Gegenbauer polynomial of degree $n$,

$$
C_{n}^{\alpha}(x)=\left((2 \alpha)_{n} / n !\right)_{2} F_{1}\left(-n, n+2 \alpha, \alpha+\frac{1}{2} ;(1-x) / 2\right),
$$

for $\alpha>0$, with ${ }_{2} F_{1}$ is the Gauss hypergeometric function.

The following analogue of the Funk-Hecke formula for $k$ -spherical harmonics will be used later on; for the proof, the reader is referred to [32]. Let $h$ be a continuous function on $[-1,1]$. Then, for any $Y_{k}^{n} \in \mathscr{H}_{k}^{n}$,

$$
\frac{1}{d_{k}} \int_{\mathbb{S}^{d-1}} V_{k}[h(\langle x, \cdot\rangle)](y) Y_{k}^{n}(y) \vartheta_{k}(y) d \sigma(y)=\Lambda_{n}(h) Y_{k}^{n}(x), x \in \mathbb{S}^{d},
$$


where $\Lambda_{n}(h)$ is a constant defined by

$$
\begin{aligned}
\Lambda_{n}(h):= & \left\{\Gamma\left(\lambda_{k}+1\right) /\left(\sqrt{\pi} \Gamma\left(\lambda_{k}+\frac{1}{2}\right)\right)\right\}\left\{n ! /\left(2 \lambda_{k}\right)_{n}\right\} \\
& \cdot \int_{-1}^{1} h(t) C_{n}^{\lambda_{k}}(t)\left(1-t^{2}\right)^{\lambda_{k}-1 / 2} d t .
\end{aligned}
$$

We summarize some basic properties of Gegenbauer polynomials in a way that we shall use later.

Fact 3 (see [[6] (1.2.10)], [[33], (3.32.3)]). For $\lambda \in \mathbb{C}$ such that $\operatorname{Re} \lambda>0$, the following two integral formulas hold:

$$
\begin{gathered}
\int_{-1}^{1}\left(1-t^{2}\right)^{\lambda-1 / 2} C_{m}^{\lambda}(t) C_{n}^{\lambda}(t) d t=\{\lambda /(n+\lambda)\} \\
\cdot\{(\sqrt{\pi} \Gamma(\lambda+1 / 2) \Gamma(2 \lambda+n)) /(n ! \Gamma(\lambda+1) \\
\cdot \Gamma(2 \lambda))\} \delta_{m, n} . \\
\int_{-1}^{1} e^{i z t}\left(1-t^{2}\right)^{\lambda-1 / 2} C_{n}^{\lambda}(t) d t=\left\{\sqrt{\pi} i^{n} \Gamma(\lambda+1 / 2)\right. \\
\cdot \Gamma(2 \lambda+n)\} /\{n ! \Gamma(2 \lambda) \Gamma(\lambda+n+1)\}(z / 2)^{n} j_{\lambda+n}(z) .
\end{gathered}
$$

Let $\mathscr{D}_{R}(\mathbb{R})^{e}$ denote the space of even compactly supported smooth functions with support in $[-R, R]$, where $R>0$. The Paley-Wiener theorem for the Hankel transform $\mathscr{H}_{\alpha}$ (see (17)) states that $\mathscr{H}_{\alpha}$ maps $\mathscr{D}_{R}(\mathbb{R})^{e}$ bijectively onto the space $\mathscr{H}_{R}(\mathbb{C})^{e}$ of even entire functions $g$ satisfying, for all $N \in \mathbb{N}$,

$$
|g(z)| \leq C_{N}(1+|z|)^{-N} e^{R|\operatorname{Im} z|}, \forall z \in \mathbb{C},
$$

for some positive constant $C_{N}$; see for instance [34].

This result has been generalized by de Jeu [23] to the Dunkl transform. To state the (complex) Paley-Wiener theorem for $\mathscr{F}_{k}$, we introduce the following notation. For $R>0$, let $\mathscr{H}_{R}\left(\mathbb{C}^{d}\right)$ be the space of entire functions $F$ on $\mathbb{C}^{d}$ with the property that for all $N \in \mathbb{N}$ there exists a constant $C_{N}>$ 0 such that

$$
|F(z)| \leq C_{N}(1+\|z\|)^{-N} e^{R\|\operatorname{Im} z\|}, \forall z \in \mathbb{C}^{d}
$$

We let $\mathscr{D}_{R}\left(\mathbb{R}^{d}\right)$ denote the space of smooth compactly supported functions with support contained in the closed ball $\overline{B(O, R)} \subset \mathbb{R}^{d}$ with radius $R>0$ and the origin as center.

Fact 4 (see [23]). The Dunkl transform $\mathscr{F}_{k}$ is a linear isomorphism between $\mathscr{D}_{R}\left(\mathbb{R}^{d}\right)$ and $\mathscr{H}_{R}\left(\mathbb{C}^{d}\right)$, for all $R>0$.

An immediate consequence of the above Paley-Wiener theorems can be stated as follows:

Lemma 5. Let $F_{0} \in C^{\infty}(\mathbb{R})^{e}$. Then, $F_{0}(\|\xi\|)=\mathscr{F}_{k} f(\xi)$ for some radial function $f$ in $\mathscr{D}_{R}\left(\mathbb{R}^{d}\right)$ if and only if $F_{0}$ extends to an entire function on $\mathbb{C}$ satisfying the estimate

$$
\left|F_{0}(z)\right| \leq C_{N}(1+|z|)^{-N} e^{R|\operatorname{Im} z|}, \forall z \in \mathbb{C},
$$

for all $N \in \mathbb{N}$.

Proof. The statement follows from the fact that $\mathscr{F}_{k} f(\xi)=$ $\mathscr{H}_{\lambda_{k}} f_{0}(\|\xi\|)$ whenever $f$ is a radial function with $f(x)=f_{0}(\|$ $x \|)$ (see (15)), together with the Paley-Wiener theorems stated above for the Hankel and the Dunkl transforms.

\section{The Range of the Spectral Projection Associated with $\Delta_{k}$}

Recall from (12) that the Dunkl transform of $f \in \mathscr{D}\left(\mathbb{R}^{d}\right)$ is defined by

$$
\mathscr{F}_{k} f(\xi)=c_{k}^{-1} \int_{\mathbb{R}^{d}} f(x) E_{k}(x,-i \xi) \vartheta_{k}(x) d x .
$$

Using polar coordinates, the Dunkl inversion formula (14) becomes

$$
\begin{aligned}
f(x) & =c_{k}^{-1} \int_{0}^{\infty} s^{2 \lambda_{k}+1} \int_{\mathbb{S}^{d-1}} \mathscr{F}_{k} f(s \eta) E_{k}(i x, s \eta) \vartheta_{k}(\eta) d \sigma(\eta) d s \\
& =\int_{0}^{\infty} s^{2 \lambda_{k}+1} P_{k}^{s} f(x) d s
\end{aligned}
$$

where

$$
P_{\mathrm{k}}^{s} f(x):=c_{k}^{-1} \int_{\mathbb{S}^{d-1}} \mathscr{F}_{k} f(s \eta) E_{k}(i x, s \eta) \vartheta_{k}(\eta) d \sigma(\eta)
$$

Notice that $\Delta_{k}^{x} P_{k}^{s} f(x)=-s^{2} P_{k}^{s} f(x)$.

From (36), we may derive a second formula for $P_{k}^{s} f$. Indeed, substituting (34) into (36), we obtain

$$
\begin{aligned}
& P_{k}^{s} f(x)=c_{k}^{-2} \int_{\mathbb{S}^{d-1}}\left(\int_{\mathbb{R}^{d}} f(y) E_{k}(-i s \eta, y) \vartheta_{k}(y) d y\right) \\
& \cdot E_{k}(i x, s \eta) \vartheta_{k}(\eta) d \sigma(\eta) \\
& =c_{k}^{-2} \int_{\mathbb{R}^{d}} f(y)(\underbrace{\int_{\mathbb{S}^{d-1}} E_{k}(\eta,-i s y) E_{k}(i s x, \eta) \vartheta_{k}(\eta) d \sigma(\eta)}_{:=I_{k}(x, y ; s)}) \vartheta_{k}(y) d y .
\end{aligned}
$$

According to [[35], page 2424], the inner integral is equal 


$$
\begin{aligned}
I_{k}(x, y ; s)= & d_{k} \sum_{n=0}^{\infty}\left\{\Gamma\left(\lambda_{k}+1\right) /\left(2^{n} \Gamma\left(\lambda_{k}+n+1\right)\right)\right\}^{2} j_{n+\lambda_{k}} \\
& (s\|x\|) j_{n+\lambda_{k}}(s\|y\|) Q_{k}^{n}(i s x,-i s y),
\end{aligned}
$$

where $d_{k}$ is the constant (25), $Q_{k}^{n}(\cdot, \cdot)$ is the reproducing kernel (26), and $j_{\alpha}$ is the normalized Bessel function (18). We now use the well-known addition formula for Bessel functions (see [[36] , p. 215]): for $a, b, \theta \in \mathbb{R}$,

$$
\begin{aligned}
j_{\alpha} & \left(\sqrt{a^{2}+b^{2}-2 a b \cos \theta}\right)=\sum_{n=0}^{\infty}\{(n+\alpha) / \alpha\} \\
& \cdot\left\{\Gamma(\alpha+1) /\left(2^{n} \Gamma(\alpha+n+1)\right)\right\}^{2}(a b)^{n} j_{n+\alpha}(a) j_{n+\alpha}(b) C_{n}^{\alpha}(\cos \theta),
\end{aligned}
$$

which converges uniformly with respect to $\theta \in \mathbb{R}$. Using (38) and (26) together with the Laplace representation (9) for $V_{k}$, we deduce that

$$
\begin{aligned}
I_{k}(x, y ; s) & =d_{k} \int_{\mathbb{R}^{d}} j_{\lambda_{k}}\left(s \sqrt{\|x\|^{2}+\|y\|^{2}-2\|y\|\langle x, \eta\rangle}\right) d \mu_{y /\|y\|}(\eta) \\
& =d_{k} \int_{\mathbb{R}^{d}} j_{\lambda_{k}}\left(s \sqrt{\|x\|^{2}+\|y\|^{2}-2\langle x, \eta\rangle}\right) d \mu_{y}(\eta) \\
& =d_{k} V_{k}\left(j_{\lambda_{k}}\left(s \sqrt{\|x\|^{2}+\|y\|^{2}-2\langle x, \cdot\rangle}\right)\right)(y) .
\end{aligned}
$$
have

Define $j_{s, \lambda_{k}}(y):=j_{\lambda_{k}}(s\|y\|)$, then, by [[35] , p. 2429], we

$$
V_{k}\left(j_{\lambda_{k}}\left(s \sqrt{\|x\|^{2}+\|y\|^{2}-2\langle x, \cdot\rangle}\right)\right)(y)=\tau_{y} j_{s, \lambda_{k}}(-x)=\tau_{-x} j_{s, \lambda_{k}}(y) .
$$

Consequently, the eigenfunction $P_{k}^{s} f$ can be rewritten as

$$
\begin{aligned}
& P_{k}^{s} f(x)=d_{k} c_{k}^{-2} \int_{\mathbb{R}^{d}} \tau_{x} f(y) j_{\lambda_{k}}(s\|y\|) \vartheta_{k}(y) d y \\
& P_{k}^{s} f(x)=2^{-\lambda_{k}} \Gamma\left(\lambda_{k}+1\right)^{-1}\left(f *_{k} j_{s, \lambda_{k}}\right)(y) .
\end{aligned}
$$

Above, we have used some of the properties of the generalized translation operator listed in Fact 2. The following statement lists the necessary conditions for Theorem 9.

Proposition 6. Assume that $f \in \mathscr{D}_{R}\left(\mathbb{R}^{d}\right)$ and let $P_{k}^{s} f(x)$ defined either by (36) or (42), with $s \in \mathbb{R}$ and $x \in \mathbb{R}^{d}$. Then the following hold:

(1) $P_{k}^{s} f(x)$ is a smooth function on $\mathbb{R} \times \mathbb{R}^{d}$

(2) $\Delta_{k}^{x} P_{k}^{s} f(x)=-s^{2} P_{k}^{s} f(x)$, where the upper index $x$ indicates the relevant variable
(3) For $x \in \mathbb{R}^{d}$ be given, $P_{k}^{s} f(x)$ extends to an even entire function of $s \in \mathbb{C}$

(4) For every $N \in \mathbb{N}$ and for every multi-index $m \in \mathbb{N}^{d}$, there exists a constant $C_{k, m, N}>0$ such that

$\left|\partial_{x}^{m} P_{k}^{s} f(x)\right| \leq C_{k, m, N}(1+|s|)^{-N} e^{(R+\|x\|)|\operatorname{Im} s|}, \forall s \in \mathbb{C}$

(5) For any $k$-spherical harmonic $Y_{k}^{\ell}$ of degree $\ell$ and for every $r>0$, the map

$$
s \mapsto s^{-2 \ell} \int_{\mathbb{S}^{d-1}} P_{k}^{s} f(r \omega) Y_{k}^{\ell}(\omega) \vartheta_{k}(\omega) d \sigma(\omega)
$$

is entire on $\mathbb{C}$.

Proof.

(1) In view of properties of the translation operator $\tau_{x}$ and the normalized Bessel function $j_{\alpha}$, the first statement follows from the representation (42) of $\mathrm{P}_{k}^{s} f(x)$.

(2) The second property is immediate from (36), since $\Delta_{k}^{x} E_{k}(x, i s \eta)=-s^{2} E_{k}(x, i s \eta)$.

(3) Now, by (42), the map $s \mapsto P_{k}^{s} f$ is certainly extends to an even entire function on $\mathbb{C}$

(4) Since $f \in \mathscr{D}_{R}\left(\mathbb{R}^{d}\right)$, it follows from the Paley-Wiener theorem for the Dunkl transform that $\mathscr{F}_{k} f$ extends to an entire function on $\mathbb{C}^{d}$ satisfying the estimate

$$
\left|\mathscr{F}_{k} f(\xi)\right| \leq C_{k, M}(1+\|\xi\|)^{-M} e^{R\|\operatorname{Im} \xi\|}
$$

for all $M \in \mathbb{N}$; see Fact 4 . Consequently, by (36), we obtain

$$
\left|\partial_{x}^{m} P_{k}^{s} f(x)\right| \leq C_{k, M}^{\prime}(1+|s|)^{-M}|s|^{|m|} e^{(R+\|x\|)|\operatorname{Im} s|}
$$

for all $M \in \mathbb{N}$, where we used the estimate $\left|\partial_{x}^{m} E_{k}(x, z)\right| \leq \| z$

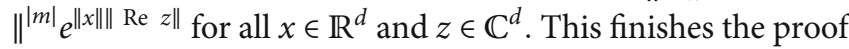
of the estimate (44).

(5) Let $Y_{k}^{\ell}$ be a $k$-spherical harmonic of degree $\ell$. By the Fubini theorem and (37), we have

$$
\begin{aligned}
\int_{\mathbb{S}^{d-1}} & P_{k}^{s} f(r \omega) Y_{k}^{\ell}(\omega) \vartheta_{k}(\omega) d \sigma(\omega) \\
\quad= & c_{k}^{-2} \int_{\mathbb{R}^{d}} f(y)\left(\int_{\mathbb{S}^{d-1}} I_{k}(r \omega, y ; s) Y_{k}^{\ell}(\omega) \vartheta_{k}(\omega) d \sigma(\omega)\right) \vartheta_{k}(y) d y,
\end{aligned}
$$


where $I_{k}(r \omega, y ; s)$ is as in (38),

$$
\begin{aligned}
I_{k}(r \omega, y ; s)= & d_{k} \sum_{n=0}^{\infty}\left\{\left(n+\lambda_{k}\right) / \lambda_{k}\right\}\left\{\Gamma\left(\lambda_{k}+1\right) /\left(2^{n} \Gamma\left(\lambda_{k}+n+1\right)\right)\right\}^{2} \\
& \cdot\left(r s^{2}\|y\|\right)^{n} j_{n+\lambda_{k}}(r s) j_{n+\lambda_{k}}(s\|y\|) V_{k}\left[C_{n}^{\lambda_{k}}(\langle\cdot, y /\|y\|\rangle)\right](\omega) .
\end{aligned}
$$

We now apply the Funk-Hecke formula (28) to deduce that

$$
\begin{aligned}
& \int_{\mathbb{S}^{d-1}} V_{k}\left[C_{n}^{\lambda_{k}}(\langle\cdot, y /\|y\|\rangle)\right](\omega) Y_{k}^{\ell}(\omega) \vartheta_{k}(\omega) d \sigma(\omega) \\
& =d_{k} \Lambda_{\ell}\left(C_{n}^{\lambda_{k}}\right) Y_{k}^{\ell}(y /\|y\|),
\end{aligned}
$$

where, by Fact 3, we have

$$
\begin{aligned}
\Lambda_{\ell}\left(C_{n}^{\lambda_{k}}\right)= & \left\{\Gamma\left(\lambda_{k}+1\right) /\left(\sqrt{\pi} \Gamma\left(\lambda_{k}+\frac{1}{2}\right)\right)\right\}\left\{\ell ! /\left(2 \lambda_{k}\right)_{\ell}\right\} \\
& \cdot \int_{-1}^{1} C_{n}^{\lambda_{k}}(t) C_{\ell}^{\lambda_{k}}(t)\left(1-t^{2}\right)^{\lambda_{k}-1 / 2} d t \\
= & \left\{\lambda_{k} /\left(\lambda_{k}+\ell\right)\right\} \delta_{\ell, n} .
\end{aligned}
$$

Using the above identities, it follows that

$$
\begin{aligned}
\int_{\mathbb{S}^{d-1}} & P_{k}^{s} f(r \omega) Y_{k}^{\ell}(\omega) \vartheta_{k}(\omega) d \sigma(\omega) \\
= & \frac{1}{2^{2 \lambda_{k}+2 \ell} \Gamma\left(\lambda_{k}+\ell+1\right)^{2}} \int_{\mathbb{R}^{d}} f(y)\left(r s^{2}\|y\|\right)^{\ell} j_{\ell+\lambda_{k}}(r s) j_{\ell+\lambda_{k}} \\
& (s\|y\|) Y_{k}^{\ell}(y /\|y\|) \vartheta_{k}(y) d y \\
= & \varphi_{\ell+\lambda_{k}}(r s) \int_{0}^{\infty} \varphi_{\ell+\lambda_{k}}(s t) f_{\ell}(t) t^{2 \lambda_{k}+1} d t
\end{aligned}
$$

where $\varphi_{\ell+\lambda_{k}}(z):=1 /\left(2^{\lambda_{k}+\ell} \Gamma\left(\lambda_{k}+\ell+1\right)\right) z^{\ell} j_{\ell+\lambda_{k}}(z)$ and

$$
f_{\ell}(t):=\int_{\mathbb{S}^{d-1}} f(t \eta) Y_{k}^{\ell}(\eta) \vartheta_{k}(\eta) d \sigma(\eta) .
$$

Above, we have used the fact that $d_{k}=c_{k} /\left\{2^{\lambda_{k}} \Gamma\left(\lambda_{k}+1\right)\right\}$; see (25). In conclusion,

$$
\begin{aligned}
s^{-2 \ell} & \int_{\mathbb{S}^{d-1}} P_{k}^{s} f(r \omega) Y_{k}^{\ell}(\omega) \vartheta_{k}(\omega) d \sigma(\omega) \\
\quad= & s^{-\ell} \varphi_{\ell+\lambda_{k}}(r s) \int_{0}^{\infty} s^{-\ell} \varphi_{\ell+\lambda_{k}}(s t) f_{\ell}(t) t^{2 \lambda_{k}+1} d t
\end{aligned}
$$

The desired result now follows from the fact that $s^{-\ell} \varphi_{\ell+\lambda_{k}}$ $(s z)$ is an entire function of $s \in \mathbb{C}$.

The following lemma is needed for later use.
(1) For any radial function $f$ on $\mathbb{R}^{d}$ with $f(x)=f_{0}(\|x\|)$ and for any $k$-spherical harmonic $Y_{k}^{\ell}$ of degree $\ell$, we have

$$
\begin{aligned}
\Delta_{k}\left(f Y_{k}^{\ell}\right)= & \left(f_{0}^{\prime \prime}(r)+\left\{\left(2 \lambda_{k}+1\right) / r\right\} f_{0}^{\prime}(r)\right. \\
& \left.-\left\{\left(\ell\left(\ell+2 \lambda_{k}\right)\right) / r^{2}\right\} f_{0}(r)\right) Y_{k}^{\ell}(\omega) .
\end{aligned}
$$

(2) Under the change of variable $f(r)=(r c)^{-\alpha} u(r / c)$, the differential equation

$$
f^{\prime \prime}(r)+(A / r) f^{\prime}(r)+\left(\mu^{2}-\left(B / r^{2}\right)\right) f(r)=0
$$

transforms to the Bessel equation $u^{\prime \prime}(r)+(1 / r) u^{\prime}(r)+(1-$ $\left.v^{2} / r^{2}\right) u(r)=0$ with

$$
\begin{aligned}
& A=2 \alpha+1, \\
& \mu=c^{-1}, \\
& B=v^{2}-\alpha^{2} .
\end{aligned}
$$

Proof.

(1) In the polar coordinates $x=r \omega$, the Dunkl Laplacian operator is expressed as

$$
\Delta_{k}=\frac{d^{2}}{d r^{2}}+\frac{2 \lambda_{\mathrm{k}}+1}{r} \frac{d}{d r}+\frac{1}{r^{2}} \Delta_{k, \mathbb{S}^{d-1}}
$$

where $\Delta_{k, \mathbb{S}^{d-1}}$ is the analogue of the Laplace-Beltrami operator on the sphere $\mathbb{S}^{d-1}$, which, in particular, has $k$-spherical harmonics as eigenfunctions,

$$
\Delta_{k, \mathbb{S}^{d-1}} Y_{k}^{\ell}(\omega)=-\ell\left(\ell+2 \lambda_{k}\right) Y_{k}^{\ell}(\omega)
$$

We refer the reader to [37] for more details on $\Delta_{k, \mathbb{S}^{d-1}}$.

(2) Obvious

Next we will list the sufficient conditions for Theorem 9.

Proposition 8. For $s \in \mathbb{R}$ and $x \in \mathbb{R}^{d}$, let $f_{s}(x)$ be a function satisfying the following conditions:

(1) $f_{s}(x)$ is smooth on $\mathbb{R} \times \mathbb{R}^{d}$

(2) $f_{s}(x)$ is an eigenfunction of the Dunkl Laplacian with eigenvalue $-s^{2}$

(3) The mapping $s \mapsto f_{s}(x)$ extends to an even entire function on $\mathbb{C}$

\section{Lemma 7.}


(4) For every $N \in \mathbb{N}$ and for every multi-index $m \in \mathbb{N}^{d}$, there exists a positive continuous increasing function $C_{k, m, N}$ on $\mathbb{R}_{+}$such that

$\left|\partial_{x}^{m} f_{s}(x)\right| \leq C_{k, m, N}(\|x\|)(1+|s|)^{-N} e^{(R+\|x\|)|I m s|}, \forall s \in \mathbb{C}$

(5) For every $r>0$ and every $k$-spherical harmonic $Y_{k}^{\ell}$ of degree $\ell$, the mapping

$$
s \mapsto s^{-2 \ell} \int_{\mathbb{S}^{d-1}} f_{s}(r \omega) Y_{k}^{\ell}(\omega) \vartheta_{k}(\omega) d \sigma(\omega)
$$

is entire on $\mathbb{C}$.

Then, there exists $f \in \mathscr{D}_{R}\left(\mathbb{R}^{d}\right)$ such that $f_{s}(x)=P_{k}^{s} f(x)$.

Proof. Define the following function

$$
f(x):=\int_{0}^{\infty} s^{2 \lambda_{k}+1} f_{s}(x) d s .
$$

The estimate (60) (with $m=0$ ) shows that the integral converge absolutely, and therefore, by assumption (1) and the estimate (60) again, $f$ is smooth on $\mathbb{R}^{d}$.

Below, we will prove that $f(x)=0$ for all $\|x\|>R$. Let $\left\{Y_{\ell, j}: 1 \leq j \leq \operatorname{dim}\left(\mathscr{H}_{k}^{\ell}\right)\right\}$ be an orthonormal basis of $\mathscr{H}_{k}^{\ell}$. By (62), we have

$$
f_{\ell, j}(r)=\int_{0}^{\infty} s^{2 \lambda_{k}+1} f_{s, \ell, j}(r) d s, r>0,
$$

where $f_{\ell, j}(r):=\left\langle f(r \cdot), Y_{\ell, j}\right\rangle_{k}$ and $f_{s, \ell, j}(r):=\left\langle f_{s}(r \cdot), Y_{\ell, j}\right\rangle_{k}$ are the $k$-spherical harmonic coefficients of $f$ and $f_{s}$, respectively. Here, $\langle\cdot, \cdot\rangle_{k}$ stands for the inner product in $L^{2}\left(\mathbb{S}^{d-1}, \vartheta_{k}(\eta) d \sigma(\eta)\right)$. To show that $f(x)=0$ for all $\|x\|>R$, it is enough to prove that $f_{\ell, j}(r)=0$ for all $r>R$. By Lemma 7 (1), the fact that $f_{s, \ell, j}(r) Y_{\ell, j}(\eta)$ are eigenfunctions of $\Delta_{k}$ with eigenvalue $-s^{2}$ implies

$$
\left\{\frac{d^{2}}{d r^{2}}+\frac{2 \lambda_{k}+1}{r} \frac{d}{d r}+\left(s^{2}-\frac{\ell\left(\ell+2 \lambda_{k}\right)}{r^{2}}\right)\right\} f_{s, \ell, j}(r)=0 .
$$

Further, by Lemma 7 (2), the solution $f_{s, \ell, j}(r)$ of equation (64) is in the following form:

$$
f_{s, \ell, j}(r)=c_{\ell, j}(s) r^{\ell} j_{\ell+\lambda_{k}}(r s)=\tilde{c}_{\ell, j}(s) r^{\ell} s^{2 \ell} j_{\ell+\lambda_{k}}(r s),
$$

where $c_{\ell, j}$ is a function which depends only on $s$, and $\tilde{c}_{\ell, j}(s)$ $:=s^{-2 \ell} \mathcal{C}_{\ell, j}(s)$. Because of the condition (5) on $f_{s}(x)$, the mapping $s \mapsto \tilde{c}_{\ell, j}(s)$ extends to an entire function of $s \in \mathbb{C}$.

On the other hand, it is known that the normalized Bessel function $j_{\alpha}(z)$ has infinitely many positive zeros $0<\rho_{\alpha}^{(1)}<$ $\rho_{\alpha}^{(2)}<\cdots$. Let $0<r_{0}<\rho_{\ell+\lambda_{k}}^{(1)}$ and define

$$
i\left(r_{0}\right):=\inf f_{z \in \mathbb{C},|z|=r_{0}}\left|j_{\ell+\lambda_{k}}(z)\right| .
$$

For $s \in \mathbb{C}$ such that $|s|>0$, the identity (65) and the assumption (4) on $f_{s}(x)$ (with $m=0$ ) imply, for $r=r_{0} /|s|$,

$$
\left|\tilde{\mathcal{C}}_{\ell, j}(s)\right| \leq C_{k, 0, N}\left(\frac{r_{0}}{|s|}\right) i\left(r_{0}\right)^{-1} r_{0}^{-\ell}|s|^{-\ell}(1+|s|)^{-N} e^{\left(R+\frac{r_{0}}{|s|}\right)|\operatorname{Im} s|},
$$

for all $N \in \mathbb{N}$. In particular, if $|s| \geq 1$, we have

$$
\left|\tilde{\mathcal{c}}_{\ell, j}(s)\right| \leq C_{k, M, r_{0}}(1+|s|)^{-M} e^{\left(R+r_{0}\right)|\operatorname{Im} s|},
$$

for all $M \in \mathbb{N}$ (recall that $C_{k, 0, N}$ is an increasing function). For the compact domain $0 \leq|s| \leq 1$, the estimate (68) holds true with a different constant. Moreover, by (65) and the evenness of $f_{s}(x)$, the map $s \mapsto \tilde{c}_{\ell, j}(s)$ is even. Applying Lemma 5, there exists a radial function $\psi \in \mathscr{D}_{R+r_{0}}\left(\mathbb{R}^{d+2 \ell}\right)$ such that $\tilde{c}_{\ell, j}(s)=$ $\mathscr{H}_{\langle k\rangle+(d+2 \ell-2) / 2} \psi_{0}(\|\xi\|)$ with $\|\xi\|=s$, where $\psi(x)=\psi_{0}(\|x\|)$ and $\mathscr{H}_{\alpha}$ is the Hankel transform. Here, we have used the fact that the Dunkl transform of radial functions at $\xi$ is a Hankel transform at $\|\xi\|$ (see (15)). Now, letting $r_{0} \longrightarrow 0$ show supp $(\psi) \subset \overline{B(O, R)}$ in $\mathbb{R}^{d+2 \ell}$.

Using again (65) and the fact that $\tilde{\mathcal{C}}_{\ell, j}(s)=\mathscr{H}_{\langle k\rangle+(d+2 \ell-2) / 2}$ $\psi_{0}(s)$, the integral (63) becomes

$$
\begin{aligned}
f_{\ell, j}(r) & =r^{\ell} \int_{0}^{\infty} \tilde{c}_{\ell, j}(s) j_{\ell+\lambda_{k}}(r s) s^{2 \ell+2 \lambda_{k}+1} d s \\
& =r^{\ell} \int_{0}^{\infty} \mathscr{H}_{\langle k\rangle+(d+2 \ell-2) / 2} \psi_{0}(s) j_{\ell+\lambda_{k}}(r s) s^{2 \ell+2 \lambda_{k}+1} d s \\
& =r^{\ell} \int_{0}^{\infty} \mathscr{H}_{\lambda_{k}+\ell} \psi_{0}(s) j_{\ell+\lambda_{k}}(r s) s^{2 \ell+2 \lambda_{k}+1} d s \\
& =2^{\lambda_{k}+\ell} \Gamma\left(\lambda_{k}+\ell\right) r^{\ell} \psi_{0}(r) .
\end{aligned}
$$

That is,

$$
\psi(x)=2^{-\left(\lambda_{k}+\ell\right)} \Gamma\left(\lambda_{k}+\ell\right)^{-1}\|x\|^{-\ell} f_{\ell, j}(\|x\|), x \in \mathbb{R}^{d+2 \ell},
$$

which implies that $f_{\ell, j}(\|x\|)=0$ for $\|x\|>R$ as desired.

It will follow that $f_{s}(x)=P_{k}^{s} f(x)$ with $f(x)=\int_{0}^{\infty} s^{2 \lambda_{k}+1} f_{s}$ $(x) d s$ provided we prove that if a function $h_{s}(x)$ satisfies assumptions (1)-(5) of Proposition 8 with $\int_{0}^{\infty} s^{2 \lambda_{k}+1} h_{s}(x) d s$ $=0$, then $h_{s}(x)=0$ for all $x \in \mathbb{R}^{d}$ and $s \in \mathbb{R}$. To prove so, it suffices to show that $\int_{0}^{\infty} s^{2 \lambda_{k}+1} h_{s, \ell, j}(x) d s=0$ implies $h_{s, \ell, j}(x)$ $=0$ for all $x \in \mathbb{R}^{d}$ and $s \in \mathbb{R}$. However, this follows by mimicking the proof given above together with the injectivity of the Hankel transform on $\mathbb{R}_{+}$.

We can now state the main result of this paper by putting the above propositions together. 
Theorem 9. Let $x \in \mathbb{R}^{d}$ and $s \in \mathbb{R}$. There exists a smooth compactly supported function $f$ with support contained in $\overline{B(O, R)}$ such that $P_{k}^{s} f(x)=f_{s}(x)$ if and only if $f_{s}(x)$ satisfies the conditions listed in Proposition 8.

The following statement illustrates an interesting application of Theorem 9. We may think of it as a spectralreformulation of the Paley-Wiener theorem for the Dunkl transform (see Fact 4).

Theorem 10. Let $\varphi(s, \eta)$ be a smooth function on $\mathbb{R} \times \mathbb{S}^{d-1}$. Then, $\varphi(s, \eta)=\mathscr{F}_{k} f(s \eta)$ for some $f \in \mathscr{D}_{R}\left(\mathbb{R}^{d}\right)$ if and only if the following two conditions hold:

(1) For each $\eta \in \mathbb{S}^{d-1}$, the map $s \mapsto \varphi(s, \eta)$ has an entire extension with the property that for all $N \in \mathbb{N}$, there exists a constant $C_{k, N}>0$ such that

$$
|\varphi(s, \eta)| \leq C_{k, N}(1+|s|)^{-N} e^{R|\operatorname{Im} s|}
$$

(2) For an arbitrary $k$-spherical harmonic $Y_{k}^{\ell}$ of degree $\ell$, the map

$$
s \mapsto s^{-\ell} \int_{\mathbb{S}^{d-1}} \varphi(s, \eta) Y_{k}^{\ell}(\eta) \vartheta_{k}(\eta) d \sigma(\eta)
$$

has an even entire extension.

Proof. Recall from (10) the Dunkl kernel $E_{k}(x, y)=V_{k}\left(e^{\langle i, y\rangle}\right)$ $(x)$. Following [38], let ${ }^{\mathrm{t}} V_{k}$ be the transpose of the Dunkl intertwining operator $V_{k}$ which satisfies on $\mathscr{D}\left(\mathbb{R}^{d}\right)$

$$
\int_{\mathbb{R}^{d}} f(x) V_{k} h(x) \vartheta_{k}(x) d x=\int_{\mathbb{R}^{d}}{ }^{\mathrm{t}} V_{k} f(x) h(x) d x .
$$

By [38], supp $(f) \subset \overline{B(O, R)}$ if and only if $\operatorname{supp}\left({ }^{\mathrm{t}} V_{k} f\right) \subset$ $\overline{B(O, R)}$.

We now proceed towards the proof of the direct part. Assume that $\varphi(s, \eta)=\mathscr{F}_{k} f(s \eta)$ for some $f \in \mathscr{D}_{R}\left(\mathbb{R}^{d}\right)$. Then,

$$
\varphi(s, \eta)=\int_{\mathbb{R}^{d}} f(x) V_{k}\left(e^{-\mathrm{is}\langle\eta \cdot\rangle}\right)(x) \vartheta_{k}(x) d x=\int_{\mathbb{R}} g_{\eta}(p) e^{-\mathrm{isp}} d p,
$$

where $g_{\eta}(p):=\int_{\langle\eta, y\rangle=p}{ }^{\mathrm{t}} V_{k} f(y) d y$. Since $\operatorname{supp}(f) \subset \overline{B(O, R)}$, it follows that $g_{\eta} \in \mathscr{D}_{R}(\mathbb{R})$. This together with the PaleyWiener theorem for the Euclidean Fourier transform on $\mathbb{R}$ completes the proof of the property (1).
Next, we turn our attention to the property (2). For $r>0$ and $s \in \mathbb{C}$, we have

$$
\begin{aligned}
\int_{\mathbb{S}^{d-1}} & P_{k}^{s} f(r \eta) Y_{k}^{\ell}(\eta) \vartheta_{k}(\eta) d \sigma(\eta) \\
= & c_{k}^{-1} \int_{\mathbb{S}^{d-1}} Y_{k}^{\ell}(\eta)\left(\int_{\mathbb{S}^{d-1}} \mathscr{F}_{k} f(s \omega) E_{k}(\omega, i r s \eta) \vartheta_{k}(\omega) d \sigma(\omega)\right) \\
\quad & \times \vartheta_{k}(\eta) d \sigma(\eta)=c_{k}^{-1} \int_{\mathbb{S}^{d-1}} \mathscr{F}_{k} f(s \omega) \\
& \times\left(\int_{\mathbb{S}^{d-1}} E_{k}(i r s \omega, \eta) Y_{k}^{\ell}(\eta) \vartheta_{k}(\eta) d \sigma(\eta)\right) \vartheta_{k}(\omega) d \sigma(\omega) .
\end{aligned}
$$

Using the Funk-Hecke formula (28) to deduce that

$$
\int_{\mathbb{S}^{d-1}} E_{k}(i r s \omega, \eta) Y_{k}^{\ell}(\eta) \vartheta_{k}(\eta) d \sigma(\eta)=d_{k} \Lambda_{\ell}\left(e^{i r s .}\right) Y_{k}^{\ell}(\omega),
$$

where, by Fact 3,

$$
\begin{aligned}
\Lambda_{n}\left(e^{i r s .}\right)= & \left\{\Gamma\left(\lambda_{k}+1\right) /\left(\sqrt{\pi} \Gamma\left(\lambda_{k}+\frac{1}{2}\right)\right)\right\} \\
& \cdot\left\{\ell ! /\left(2 \lambda_{k}\right)_{\ell}\right\} \int_{-1}^{1} e^{i r s t} C_{\ell}^{\lambda_{k}}(t)\left(1-t^{2}\right)^{\lambda_{k}-1 / 2} d t \\
= & \left\{\Gamma\left(\lambda_{k}+1\right) /\left(2^{\ell} \Gamma\left(\lambda_{k}+\ell+1\right)\right)\right\}(\text { irs })^{\ell} j_{\ell+\lambda_{k}}(r s) .
\end{aligned}
$$

This shows that

$$
\begin{aligned}
\int_{\mathbb{S}^{d-1}} & P_{k}^{s} f(r \eta) Y_{k}^{\ell}(\eta) \vartheta_{k}(\eta) d \sigma(\eta) \\
= & \left\{1 /\left(2^{\lambda_{k}+\ell} \Gamma\left(\lambda_{k}+\ell+1\right)\right)\right\}(i r s)^{\ell} j_{\ell+\lambda_{k}}(r s) \\
& \times \int_{\mathbb{S}^{d-1}} \mathscr{F}_{k} f(s \omega) Y_{k}^{\ell}(\omega) \vartheta_{k}(\omega) d \sigma(\omega) .
\end{aligned}
$$

That is,

$$
\begin{aligned}
s^{-2 \ell} \int_{\mathbb{S}^{d-1}} P_{k}^{s} f(r \eta) Y_{k}^{\ell}(\eta) \vartheta_{k}(\eta) d \sigma(\eta) & =\left\{1 /\left(2^{\lambda_{k}+\ell} \Gamma\left(\lambda_{k}+\ell+1\right)\right)\right\}(i r)^{\ell} j_{\ell+\lambda_{k}}(r s) \\
& \times s^{-\ell} \int_{\mathbb{S}^{d-1}} \varphi(s, \omega) Y_{k}^{\ell}(\omega) \vartheta_{k}(\omega) d \sigma(\omega) .
\end{aligned}
$$

Since $s \mapsto P_{k}^{s} f$ and $s \mapsto j_{\ell+\lambda_{k}}(r s)$ are even, it follows that the map $s \mapsto s^{-\ell} \int_{\mathbb{S}^{d-1}} \varphi(s, \omega) Y_{k}^{\ell}(\omega) \vartheta_{k}(\omega) d \sigma(\omega)$ is even. The analycity of the latter map is immediate from Preposition 6.

For the converse part, define

$$
f_{s}(x):=c_{k}^{-1} \int_{\mathbb{S}^{d-1}} \varphi(s, \eta) E_{k}(i x, s \eta) \vartheta_{k}(\eta) d \sigma(\eta), s \in \mathbb{R}, x \in \mathbb{R}^{d} .
$$


By the assumptions (1) and (2) on $\varphi(s, \eta)$ and (11), the function $f_{s}$ satisfies the hypothesis of Theorem 9. Thus, there exists $f \in \mathscr{D}_{R}\left(\mathbb{R}^{d}\right)$ such that $f_{s}(x)=P_{k}^{s} f(x)$. That is,

$$
c_{k}^{-1} \int_{\mathbb{S}^{d-1}}\left\{\varphi(s, \eta)-\mathscr{F}_{k} f(s \eta)\right\} E_{k}(i x, s \eta) \vartheta_{k}(\eta) d \sigma(\eta)=0,
$$

which implies

$$
c_{k}^{-1} \int_{0}^{\infty} \int_{\mathbb{S}^{d-1}}\left\{\varphi(s, \eta)-\mathscr{F}_{k} f(s \eta)\right\} E_{k}(i x, s \eta) \vartheta_{k}(\eta) d \sigma(\eta) s^{2 \lambda_{k}+1} d s=0 .
$$

This finishes the proof of the converse part.

\section{Data Availability}

No data were used to support this study.

\section{Conflicts of Interest}

The authors declare that they have no conflicts of interest.

\section{Acknowledgments}

S. Ben Said would like to thankfully acknowledge the financial support awarded by UAEU through the Start-up grant. No. G00002950.

\section{References}

[1] C. F. Dunkl, "Differential-difference operators associated to reflection groups," Transactions of the American Mathematical Society, vol. 311, no. 1, pp. 167-183, 1989.

[2] B. Amri, J.-P. Anker, and M. Sifi, "Three results in Dunkl analysis," Colloquium Mathematicum, vol. 118, no. 1, pp. 299-312, 2010.

[3] J.-P. Anker, N. B. Salem, J. Dziuban'ski, and N. Hamda, “The Hardy space $\mathrm{H}^{1}$ in the rational Dunkl setting," Constructive Approximation, vol. 42, pp. 93-128, 2015.

[4] O. Chalykh, "Quantum Lax pairs via Dunkl and Cherednik operators," Communications in Mathematical Physics, vol. 369, no. 1, pp. 261-316, 2019.

[5] F. Dai and Y. Xu, "Analysis on h-harmonics and Dunkl transforms," in Advanced Courses in Mathematics, S. Tikhonov, Ed., CRM Barcelona. Birkhüser/Springer, Basel, 2015.

[6] F. Dai and Y. Xu, Approximation Theory and Harmonic Analysis on Spheres and Balls, Springer Monographs in Mathematics, Springer, New York, 2013.

[7] J. Dziubanski and A. Hejna, "Hörmander's multiplier theorem for the Dunkl transform," Journal of Functional Analysis, vol. 277, no. 7, pp. 2133-2159, 2019.

[8] L. Deleaval, M. Kemppainen, and C. Kriegler, "Hörmander functional calculus on UMD lattice valued $\mathrm{L}^{\mathrm{p}}$ spaces under generalized Gaussian estimates," https://arxiv.org/abs/1806 .03128 .

[9] L. Gallardo and C. Rejeb, “A new mean value property for harmonic functions relative to the Dunkl-Laplacian operator and applications," Transactions of the American Mathematical Society, vol. 368, no. 5, pp. 3727-3753, 2016.
[10] D. V. Gorbachev, V. I. Ivanov, and S. Y. Tikhonov, "Positive $L^{p}$ -bounded Dunkl-type generalized translation operator and its applications," Constructive Approximation, vol. 49, no. 3, pp. 555-605, 2019.

[11] M. F. E. de Jeu, "The Dunkl transform," Inventiones Mathematicae, vol. 113, no. 1, pp. 147-162, 1993.

[12] H. Mejjaoli and K. Trimèche, "On a mean value property associated with the Dunkl Laplacian operator and applications," Integral Transforms and Special Functions, vol. 12, no. 3, pp. 279-302, 2001.

[13] E. M. Opdam, "Dunkl operators, Bessel functions and the discriminant of a finite Coxeter group," Compositio Mathematica, vol. 85, pp. 333-373, 1993.

[14] M. Rösler, "Generalized Hermite polynomials and the heat equation for Dunkl operators," Communications in Mathematical Physics, vol. 192, no. 3, pp. 519-542, 1998.

[15] S. Thangavelu and Y. Xu, "Convolution operator and maximal function for the Dunkl transform," Journal d'Analyse Mathématique, vol. 97, no. 1, pp. 25-55, 2005.

[16] W. O. Bray, "A spectral Paley-Wiener theorem," Monatshefte für Mathematik, vol. 116, no. 1, pp. 1-11, 1993.

[17] W. O. Bray, "Generalized spectral projections on symmetric spaces of noncompact type: Paley-Wiener theorems," Journal of Functional Analysis, vol. 135, no. 1, pp. 206-232, 1996.

[18] A. D. Ionescu, "On the Poisson transform on symmetric spaces of real rank one," Journal of Functional Analysis, vol. 174, no. 2, pp. 513-523, 2000.

[19] J. Jana, "Image of the Schwartz space under spectral projection," Mathematische Zeitschrift, vol. 270, no. 3-4, pp. 589$611,2012$.

[20] S. Koizumi, "An analogue of the spectral projection for homogeneous trees," Hiroshima Mathematical Journal, vol. 43, no. 2, pp. 207-221, 2013.

[21] E. K. Narayanan and S. Thangavelu, "A spectral Paley-Wiener theorem for the Heisenberg group and a support theorem for the twisted spherical means on $\mathbb{C}^{\mathrm{n}}$," Annales de l'institut Fourier, vol. 56, pp. 459-473, 2006.

[22] R. S. Strichartz, "Harmonic analysis as spectral theory of Laplacians," Journal of Functional Analysis, vol. 87, no. 1, pp. 51-148, 1989.

[23] M. F. E. de Jeu, "Paley-Wiener theorems for the Dunkl transform," Transactions of the American Mathematical Society, vol. 358, no. 10, pp. 4225-4251, 2006.

[24] C. F. Dunkl, "Reflection groups and orthogonal polynomials on the sphere," Mathematische Zeitschrift, vol. 197, no. 1, pp. 33-60, 1988.

[25] M. Rösler, "Positivity of Dunkl?s intertwining operator," Duke Mathematical Journal, vol. 98, no. 3, pp. 445-463, 1999.

[26] K. Trime'Che, "Paley-Wiener theorems for the Dunkl transform and Dunkl translation operators," Integral Transforms and Special Functions, vol. 13, no. 1, pp. 17-38, 2002.

[27] C. F. Dunkl, "Integral kernels with reflection group invariance," Canadian Journal of Mathematics, vol. 43, no. 6, pp. 1213-1227, 1991.

[28] C. F. Dunkl, "Hankel transforms associated to finite reflection groups," Contemporary Mathematics, vol. 138, pp. 123-138, 1992.

[29] C. F. Dunkl and Y. Xu, "Orthogonal Polynomials of Several Variables," in Encyclopedia of Mathematics and its Applications, Cambridge University Press, Cambridge, 2nd edition, 2014. 
[30] S. Ben Saïd and B. Ørsted, "The wave equation for Dunkl operators," Indagationes Mathematicae, vol. 16, no. 3-4, pp. 351391, 2005.

[31] $\mathrm{Y} . \mathrm{Xu}$, "Integration of the intertwining operator for $\mathrm{h}$ harmonic polynomials associated to reflection groups," Proceedings of the American Mathematical Society, vol. 125, no. 10, pp. 2963-2974, 1997.

[32] Y. Xu, "Funk-Hecke formula for orthogonal polynomials on spheres and on balls," The Bulletin of the London Mathematical Society, vol. 32, no. 4, pp. 447-457, 2000.

[33] G. N. Watson, "A Treatise on the Theory of Bessel Functions, Cambridge Mathematical Library," Cambridge University Press, Cambridge, 1995.

[34] T. Koornwinder, "A new proof of a Paley-Wiener type theorem for the Jacobi transform," Arkiv för Matematik, vol. 13, no. 1-2, pp. 145-159, 1975.

[35] M. Rösler, "A positive radial product formula for the Dunkl kernel," Transactions of the American Mathematical Society, vol. 355, no. 6, pp. 2413-2439, 2003.

[36] G. Andrews, R. Askey, and R. Roy, Special Functions, Encyclopedia of Mathematics and its Applications, 71, Cambridge University Press, Cambridge, 1999.

[37] Y. Xu, "Uncertainty principle on weighted spheres, balls and simplexes," Journal of Approximation Theory, vol. 192, pp. 193-214, 2015.

[38] K. Trimèche, "The Dunkl intertwining operator on spaces of functions and distributions and integral representation of its dual," Integral Transforms and Special Functions, vol. 12, no. 4, pp. 349-374, 2001. 